\title{
Sur l'Evolution du Cartilage de Meckel d'une Chauve-souris Frugivore, Epomops franqueti strepitans Andersen (Macrochiroptera, Pteropodidae)
}

\author{
par
}

M. FRIANT*

Le Cartilage de Meckel correspond, dans la série des Vertébrés, à la partie antérieure du premier arc viscéral des Poissons cartilagineux, chez lesquels il persiste toute la vie.

Les présentes recherches ont trait au Cartilage de Meckel d'un Chiroptère frugivore des régions tropicales d'Afrique, Epomops franqueti strepitans Andersen. L'exemplaire étudié est un foetus avancé, rapporté de la Côte d'Ivoire, en 1954, par M. V. AELlEN et mis aimablement à ma disposition par M. M. les Docteurs E. DotTREns, Directeur, et M. V. AEllen, Conservateur, du Museum d'Histoire Naturelle de Genève.

Les coupes pratiquées du côté gauche de la tête sont parasagittales et numérotées de dedans en dehors, à partir du plan médian.

Sur la coupe IV, vers l'avant de la mâchoire inférieure, au niveau des sacs dentaires des incisives centrales (temporaire et définitive), la section du Cartilage de Meckel, bien visible, est arrondie en arrière. Son ossification se produit de bas en haut. Il existe (fig. 1) :

1) Une zone de cartilage hyalin normal, avec quelques chondroblastes en voie de division. La substance fondamentale est abondante, le périchondre, distinct.

2) Une zone de cartilage hypertrophié : les chondroblastes augmentent de volume, se détachent de leurs capsules, plus ou moins polyédriques, très minces, qui deviennent de simples cloisons de séparation, la sustance fondamentale ayant presque disparu. De nombreux noyaux cellulaires sont effacés.

3) Une zone d'ossification (fig. 1 et 2): les capsules cartilagineuses s'ouvrent les unes dans les autres et forment de vastes lacunes irrégulières, festonnées, séparées par les restes des capsules cartilagineuses, qui semblent s'ossifier. Des cellules embryonnaires remplissent les lacunes; elles édifient la substance osseuse et constitueront les ostéoblastes.

4) Au-delà de la zone lacunaire, l'os spongieux est formé.

En somme, au cours de la calcification, les cellules et la substance fondamentale du cartilage disparaissent en presque totalité, quelques fragments capsulaires étant, seuls, calcifiés.

Comme il est de règle, la calcification des autres centres cartilagineux de la tête

* Professeur à l'Ecole d'Anthropologie, Paris et Membre Titulaire du Groupement International pour la Recherche Scientifique en Stomatologie (G. I. R. S.).

Adresse de l'auteur: Docteur M. Friant, chez Madame Cremont, 103 rue Louis Rouquier, Levallois, Seine, France. 
(encore peu visibles, à ce stade) est la même que celle du Cartilage de Meckel.

Cette évolution est comparable à l'ossification du cartilage de conjugaison des os longs, chez les Mammifères et l'Homme, mis à part que le processus est très diffus.

Chez un jeune Chiroptère insectivore de nos régions (MiCROCHIROPTERA, Myotis myotis (Borkh.)), (M. FRIANT, 1964), il existe, outre les zones marquées ici, une zone de vascularisation intense, entre le cartilage hypertrophié et la zone d'ossification lacu-

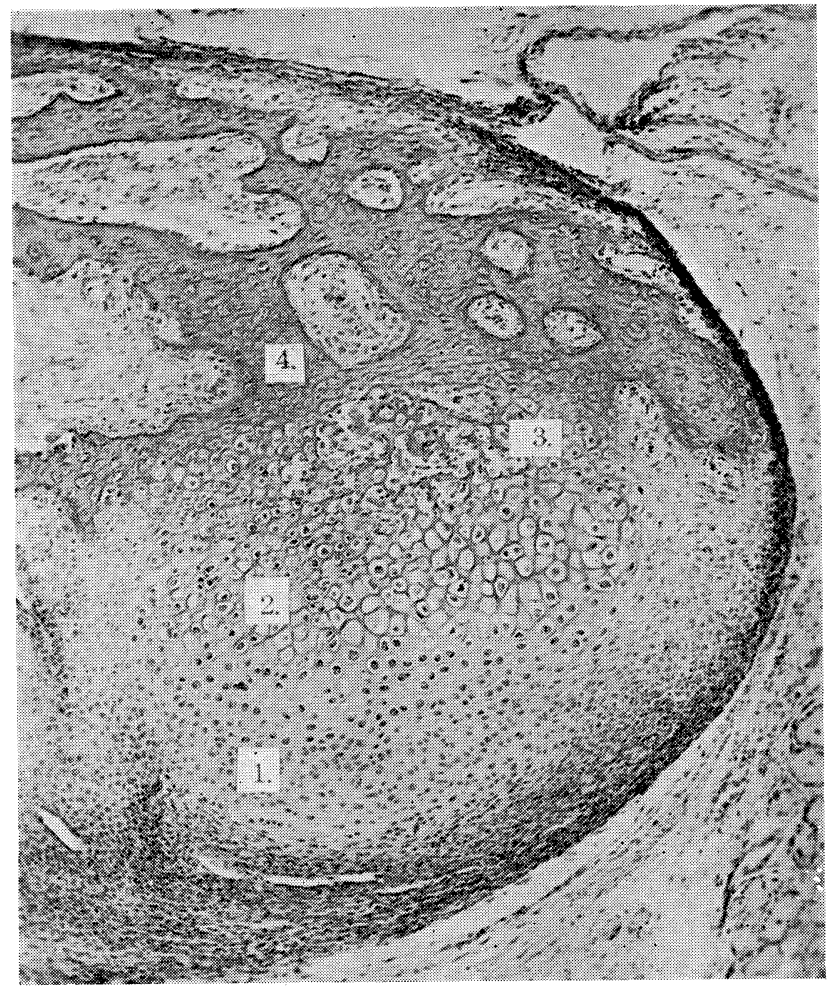

Fig. 1

Epomops franqueti strepitans Andersen (Macrochiroptera, Pteropodidae). Foetus avancé. Adipodoumé/Abidjan. Mission V. AELlen en Côte d'Ivoire, 1954, No 923 Coll. Museum Genève. Coupe IV, parasagittale, de la tête, dans sa partie antérieure. Le Cartilage de Meckel, en cours d'évolution, est figuré. - L'avant est à gauche, la partie supérieure, en haut.

1. zone de cartilage hyalin normal.

2. zone de cartilage hypertrophié.

3. zone d'ossification.

4. os spongieux.

80 fois grandeur naturelle environ.

Coupe du Centre de Cytologie de Genève.

Photographie de l'Institut de Pathologie de Genève.

naire. De plus, une zone ostéogène vasculaire, avec quelques cellules géantes et de nombreuses cellules embryonnaires, sans trace de tissu cartilagineux ni osseux, est visible en arrière de la zone d'ossification.

Ainsi que je l'ai déjà souligné, l'évolution de Cartilage de Meckel et des centres 
cartilagineux du crâne varie considérablement avec les groupes mammaliens considérés. L'ossification décrite plus haut chez les CHIROPTERA : enchondrale, avec envahissement de cellules embryonnaires, est caractéristique de cet Ordre. - Par contre, cette ossification est conjonctive, chez les INSECTIVORES primitifs, enchondrale directe, chez les RONGEURS et l'HOMME.

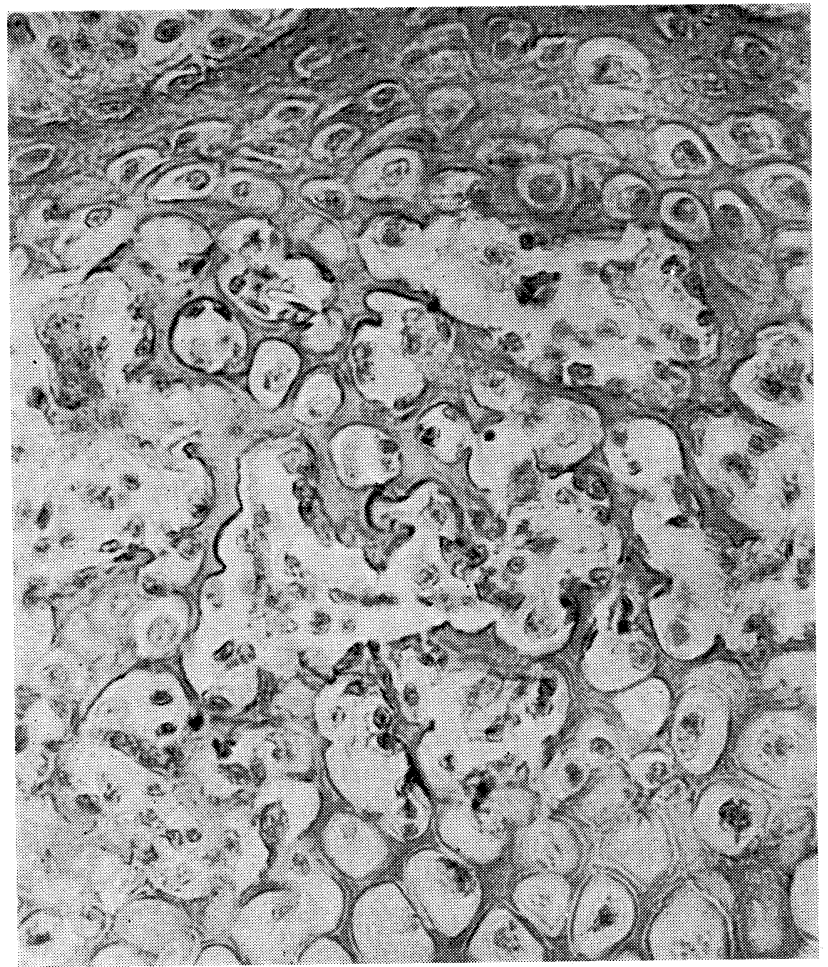

Fig. 2

Epomops franqueti strepitans Andersen. Même coupe que figure 1: une partie de la zone d'ossification (3) grossie 320 fois environ.

C'est, peut-être, en raison de la vie aérienne des Chauves-Souris que les cartilages de leur tête s'ossifient d'une manière fort différente et plus résistante que les cartilages de la tête des autres Mammifères et de l'Homme; chez ces derniers, seuls, les cartilages des os longs du corps subissent une évolution comparable.

Je remercie vivement toutes les personnes qui m'ont facilité cette étude, en particulier M. le Docteur G. Riotton et M. le Professeur E. Rutihauser, de Genève.

\section{Résumé}

Le Cartilage de Meckel de l'Epomops, Chauve-Souris frugivore (MACROCHIROPTERA) d'Afrique tropicale, subit une ossification enchondrale comparable à l'ossification des autres centres cartilagineux de la tête, comme il est de règle. L'évolution est la suivante : 
a) Les chondroblastes augmentent de volume, se détachent de leurs capsules devenues polyédriques, dégénèrent et s'effacent, tandis que la substance fondamentale disparaît.

b) Des lacunes, entourées de fragments de capsules, se forment et sont envahies par des cellules embryonnaires.

c) Les cellules embryonnaires édifient la substance osseuse, puis se transforment en ostéoblastes.

Cette évolution, proche de celle du Cartilage de Meckel des Chauves-Souris insectivores (MICROCHIROPTERA), est caractéristique de l'Ordre entier des Chiroptera.

\section{English Summary}

\section{On the evolutiom of the Meckel's Cartilage in a frugivorous Bat, Epomops franqueti strepitans Andersen \\ (Macrochiroptera, Pteropodidae)}

The Meckel's Cartilage of Epomops, a fructivorous bat (Macrochiroptera) of tropical Africa, undergoes an intracartilaginous ossification, like its head's other cartilaginous centers, as it is the rule. The evolution is as follows :

a) The chondroblasts become voluminous, separated from their capsules (polyedral now), degenerated and effaced, while the fundamental matrix disappears.

b) The gaps, surrounded with capsular fragments, are made up and invaded by embryonal cells. blasts.

c) The embryonal cells set up the osseous matrix and, afterwards, become osteo-

This evolution, near the one of the Meckel's Cartilage in insectivorous bats (Microchiroptera), is characteristic of the Chiroptera Order in its whole.

\section{Références}

M. FRIANT: Sur les premiers stades d'ossification du Cartilage de Meckel (Rongeurs). - Acta anat. 32 : 100 (1958).

Ditto : Sur l'évolution du Cartilage de Meckel (Insectivores). - Acta anat. 34 : 292 (1958).

Ditto : Sur l'ossification enchondrale du Cartilage de Meckel chez les Rongeurs. - Bull. Group. Int. Rech. Sc. Stomat. 2 : 7 (1959).

Ditto : L'evolution du Cartilage de Meckel humain, jusqu'à la fin du sixième mois de la vie foetale. - Acta anat. 41: 228 (1960).

Ditto : Sur l'ossification du Cartilage de Meckel d'une Chauve-Souris, le Grand Murin (Microchiroptera, Myotis myotis (Borkh.)). - Acta anat. 57 : 66 (1964). 